\title{
Predictive Fail-Safe \\ Improving the Safety of Industrial Environments through Model-based Analytics on hidden Data Sources
}

\author{
Amer Kajmakovic*, Robert Zupanc ${ }^{\dagger}$, Simon Mayer*, Nermin Kajtazovic ${ }^{\dagger}$, Martin Hoeffernig ${ }^{\dagger}$ and Herwig Vogl $^{\dagger}$ \\ *Pro2Future GmbH and Graz University of Technology, Graz, Austria \\ †Siemens AG Österreich, Graz, Austria \\ Email: amerkajmakovic@pro2future.at
}

\begin{abstract}
This paper explores how the functional safety of industrial deployments can be improved through emerging Industrie 4.0 approaches. We discuss how new sources of data, that are becoming accessible through advancing digitalization, can be used for this purpose, and how principles from predictive maintenance systems can be applied to industrial fail-safe applications: based on data from the industrial components themselves and from their environment as well as on metadata about interactions between these systems and people, we propose to create a model-based monitoring and controlling system that focuses on preserving the functional safety of the installation as a whole. We expect such a Predictive Fail-Safe system to mitigate or even prevent unsafe consequences of failures even in highly dynamic "smart factories", thereby reducing or preventing harm to other equipment, the environment, and the involved people.
\end{abstract}

\section{Introduction}

With the proliferation of industrial monitoring and control systems over the past few decades, a huge amount of data has been produced. At the very beginning, this data was used as part of explicit weekly or monthly technical checks. Over time, awareness of the importance of processing and analyzing the obtained data increased, and with the rapid advancement of machine learning and communication technologies, data got a significant role: industry innovators have realized that we can use this data to significantly improve the flexibility, efficiency, and reliability of industrial installations. Looking to the future, Cisco predicts there will be 50 billion devices connected to the Internet by 2020 [1], a large part of them generating data in industrial settings that will be used to optimize industrial processes and to increase their flexibility and integration along the product life cycle.

As the Industrie 4.0 paradigm is spreading, we need to take into consideration additional aspects of industrial processes, such as their safety. The desire for better and faster production has created an industrial environment where people and machines collaborate in the same physical space and without separation [2]. Consequently, the safety of people and of the equipment emerged as one of the biggest concerns for engineers in human-robot collaborative work settings. Here, it is crucial to note that there is a clear recognition that the role of people in the production will never be redundant: in fact, industrial outfits that implement Industrie 4.0 systems stress that people remain the key players ${ }^{1}$.

Also from the domain of industrial fail-safe, in recent years manufacturers of Programmable Logic Controllers (PLCs) have invested a lot of resources in upgrading their PLCs so that they are capable to detect anomalies and to ensure safety for the system and workers when failure happens. Still, complex systems with multiple control units still represent a significant challenge, since the optimal safe state of the entire system needs to be determined (and reached in time and space). In these cases, a simpler solution often is to put the entire system in a safe state, but this means that many components of an installation need to be degraded in their function, which is costly since it might imply that the entire process might come to a halt.

For improving functional safety in industrial environments, we suggest that current analysis tools from the domain of predictive analytics should be applied to help us identify sources of safety-relevant data, and to extract actionable information from them: modern machine learning and artificial intelligence approaches, in combination with a reliable data collection and storage infrastructure, can be applied in these settings [3], [4], but for achieving the best possible results, we need more reliable data sources that are relevant to industrial functional safety.

\section{Background and Related Work}

Industrial data is currently mostly used for analyzing operational and maintenance-relevant data, in particular in the context of predictive maintenance ( $\mathrm{PdM})$ systems. In conventional PdM, data from control and self-diagnostics are used for predicting possible failures and good results have been obtained even with limited amounts of data [5]. One of the quickest wins for PdM has been in the industrial sector, where data from more and more devices is gathered and model-based condition monitoring is applied to uncover warning signs of costly failures before they occur: for example, manufacturers of valves and automation components continuously monitor the performance of manufacturing

1. See https://www.boschrexroth.com/en/xc/trends-and-topics/industry4-0/internet-of-things/internet-of-things-1 
processes to detect and replace deteriorating components before they fail, improving overall efficiency [6].

For further improvements in the field of industrial safety, companies that adopt Industrie 4.0 principles will need to integrate approaches from PdM and from safety analysis. To do this, it is necessary to analyze four types of scenarios regarding safety-relevant system failures:

(a) A failure is predicted and can be prevented without switching (sub)systems to the safe state (e.g., by early servicing)

(b) A failure is predicted but cannot be prevented without switching (sub)systems to the safe state

(c) A failure is not predicted, and (sub)systems are immediately switched to the safe state when it occurs

(d) A failure is not predicted nor handled.

Scenario a) is the main concern of PdM. This is the least painful scenario and it does not endanger the safety of people or of the system. Often, early servicing of the system can avoid incurring large costs due to system failure.

Scenario b) represents a combination of the PdM and fail-safe responses and the main focus of this paper: in this scenario, an analytics system detects that a failure might be about to happen but cannot be prevented. Thus, the analytics system must inform the fail-safe components that they need to be prepared to ensure the safety of the (sub)system and of involved people. In this case, time is one of the main factors: the system should be rapidly configured with the relevant information to swiftly switch to the safe state if the failure actually occurs, to avoid any further damage than necessary. Crucially the ability to take predictions about failsafe actions drastically increases its time budget for deciding precisely which fail-safe actions to take (e.g., when dealing with complex setups) and to weigh economic considerations (e.g., a complete stop of the system might be feasible).

Scenario c) relies exclusively on the fail-safe system. This system should be able to detect the issue and to switch the entire system (or selected subsystems) to the safe state. In this case, the cost of putting the system back into a working order and of repairing any underlying and collateral problems is usually larger than in the other two cases. Also, there is a higher probability that people will be injured, and that the system might get damaged because the failure is not expected in this case.

Scenario d) is the worst-case scenario. Appearances of this uncontrolled situations could have devastating consequences for industrial (sub)system and his surroundings, including people.

The ability to make predictions about the safety state of an industrial system represents a highly desirable feature of Industrie 4.0 systems: especially for scenario b), the meaningful integration of PdM with fail-safe concepts could prevent harm to workers and equipment, help to better target fail-safe measures, and reduce the maintenance effort for system components.

\section{Predictive Fail-Safe}

In our research, we investigate how the suggested predictive approach fits with current approaches and systems for fail-safe operation in the context of future smart factories. First, we aim to improve the safety behavior of these systems by tapping into remaining data sources, in particular from industrial fail-safe modules. Second, we expect that the traditional, static, approach to fail-safe operation will need to be adapted to dynamic environments, as smart factories are expected to exhibit a great deal of dynamicity, involving rapid changes of tooling, the physical movement of robots, and even the reconfiguration of entire manufacturing processes [7] [8] - consequently, future fail-safe systems should adapt to or, ideally, anticipate these dynamics in order to guarantee fail-safe properties at all times. To do this, we are working on the integration of PdM and fail-safe operation, which we refer to as Predictive Failsafe $(P d F)$ : the ability of a system to adapt its fail-safe measures to new configurations and situations that dynamically arise in smart factory environments with the goal of protecting itself and its working environment, including human workers.

Similar to PdM, PdF systems rely on two main elements: data sources for obtaining data that is relevant to failsafe predictions, and prediction algorithms for analyzing the collected data. Identifying safety-relevant data sources and obtaining access to their data is not straightforward, since fail-safe components are usually shielded from other components of an automation system. Obtaining this data is crucial to PdF however, especially since insufficient data quality can lead to wrong conclusions and decisions, which is especially critical when dealing with safety-relevant data. On the other hand, we expect that it should be readily possible to adapt current machine learning systems to PdF, once reliable sources of safety-relevant data have been identified.

In the rest of this paper, we first discuss these data sources and provide a classification that should inform their exploitation in the context of PdF systems (Section 3.1) and then discuss two complementary approaches to modeling fail-safe systems based on these data sources in (Section 3.2)

\subsection{Data sources}

From our perspective, data sources for industrial failsafe analytics that would be valuable for PdF systems can be divided into two main groups (see Fig. 1 for an overview). The first group are data sources that are directly connected to the safety devices or systems, which we refer to as Component Data. The second group of data sources is not strictly connected to the industrial system, but is collected from the interaction of the system with its environment; hence we refer to this group as Environmental Data.

3.1.1. Component Data. This group includes process control and self-diagnostic data from the devices, which are already known and widely used in safety analysis.

A new type of data source that we suggest to add to this group is data that can be derived from operational 


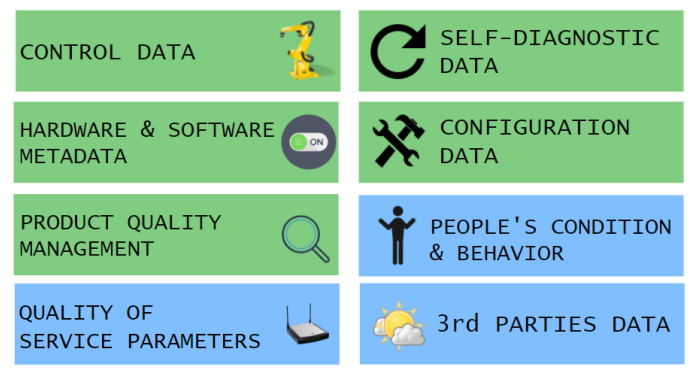

Figure 1. Overview of potentially valuable data sources for a PdF system.

data, but is not directly relevant on the operational level (and, consequently, is usually not recorded). Examples of this kind of data are device and process metadata streams both related to hardware processes (e.g., number of presses of an emergency shut-down button) and software processes (e.g., number of restarts of a self-test), as well as data about underlying physical quantities of the system (e.g., supply voltage of a component). Consider a process control system that is usually working, but each month there is a need for one of its components to be restarted because of an unknown reason. On the monthly base or on the system level, this situation does not look dangerous, however it would be very relevant to be able to learn and predict whether the average time between restarts is converging or diverging. Likewise, correlations between these restarts and the behavior of other components of the system should be exposed and investigated. Collecting data about the restart behavior of the component could also be useful for identifying the root cause of the problem itself. Similar non-operational data can be obtained from almost every device and should be considered as a new source of data within PdF systems.

Another example of the data source that is present, but rarely used is the configuration data of the devices. From the configuration of devices, we can extract useful information about the behavior of the system. For example, if the operator is reconfiguring a device or system much more often than usually, this could be an indication that something is not working properly.

The third Component Data source that should be considered in the context of PdF is data from product quality measurements. This data is currently mainly used as a source of feedback for further improvements of a product. However, we can also use quality measurements to gather information that can be useful to the safety analysis: for instance, deviations between a products design and the finalized product might indicate problems in the manufacturing process that are potentially relevant to the systems safety (and might also be valuable for PdM systems).

3.1.2. Environmental Data. This group refers to data that reflects the behavior of the system's environment and the interaction of the system with its environment, where we specifically consider people and their behavior as part of the environment. The increasing digitalization of factories and of the people within them, and the provisioning of environmental data via open interfaces in the "cloud" enables us to use these pools of data for PdF systems. This means that, similar to PdM, any kind of (trusted) data that our system can obtain can in principle be used as input data for fail-safe analysis, prediction, and control: data such as the surrounding temperature, pressure, humidity, electromagnetic radiation, etc. might prove particularly valuable for this purpose.

Another data source that is not directly connected with the system but can provide relevant information is the behavior of people who physically or virtually interact with the system: operators who work in close physical proximity to the devices could be monitored and we can collect data on their current condition (e.g., to identify the work they are currently doing, but also regarding medical data such as their heart rate or blood pressure). Less private data such as observations of sudden movements (e.g., if a worker is startled by a particular noise), gestures, or loud screams might be particularly valuable for a PdF (or PdM) system, as these might be connected to the state of surrounding industrial equipment. Likewise, virtual interactions of people with industrial systems, e.g. via control or configuration interfaces, can be recorded and analyzed to predict fail-saferelevant properties of the system.

Finally, we can derive safety-relevant information also from interaction patterns among devices. For instance, the number, rate, and burstiness (i.e., the Fano factor) of transmitted messages between two devices can yield clues about potential problems with one of the devices, or a different part of the system. Also, communication metadata that can be extracted from the devices, such as the number of lost packets and other quality-of-service parameters (e.g., from communication protocols such as PROFIsafe), can be extracted and used for further analysis.

\subsection{Modeling for Predictive Failsafe Systems}

Combining the data sources discussed above with traditional data from process control and self-diagnostics as well as with operational data gives rise to the creation of powerful condition monitoring and control models for the purpose of PdF. We suggest and discuss two types of such models that we expect might prove particularly useful in the industrial fail-safe domain:

The first type is a Local System Model (LSM), that is derived exclusively from local data and is thus tailored to a specific instance of an industrial system. The LSM is usually not shared with other systems and might be stored on a local machine or a private local cloud. These models are widely applicable, even when there is only a single deployed instance of a specific automation system: for example, through our research, we have encountered a one-of-a-kind car seat manufacturing system that had to be created from scratch and tailored to a specific setting in this case, there is no need for the data obtained from the system to leave the premises. Since the modeling and evaluation takes place on the local infrastructure, this should also contribute to speeding up the PdF process. 
The second type of model that we envision is a Global System Model (GSM). This model is shared across several (sufficiently similar) industrial systems and is trained using data from all these systems. GSMs are expected to offer a broader range of features based on their ability to draw conclusions across installations of similar automation systems in varying environments. Based on a GSM, a PdF system could for instance learn the optimal configuration of a specific failsafe system across a range of environmental settings (e.g., environments with low electromagnetic radiation vs. harsh EM environments) and produce suggestions for operators to adapt their systems accordingly - and the same information can be used as the basis of a recommender system for configuring green-field deployments. On the downside, using GSMs implies that the model is stored remotely, and all systems that contribute to and use it must have access to the remote servers, which increases security concerns. Also, care must be taken regarding incomplete and incompatible data about the environmental conditions of different deployments that contribute to a GSM - often, scaling or norming of values will be necessary to obtain valid results.

Finally, a combination of both types of model can be used to derive generic models of specific fail-safe components across all their usage scenarios. This generic model could be used as input for more realistic simulations, for instance as part of end-of-line tests. In addition, comparing the generic model of a fail-safe component to its individual LSMs might reveal safety-relevant deviations that are due to the specific deployment environment of a component.

\subsection{Challenges}

Continuing our work on PdF systems for industrial automation, we expect our biggest challenge to be associated with obtaining sufficient amounts of relevant data from the discussed sources and ensuring the data quality, which is crucial for the application of supervised learning approaches in this context. To train our PdF systems, we require data that exhibits safety-relevant anomalies and failures. We thus plan to manually create controlled anomalies in monitored systems to obtain fail-safe data for these situations - creating such anomalies will not be easy, sometimes even impossible, especially if the system is already running productively. We will furthermore attempt to obtain fail-safe information from several different systems, as this will increase our chance of recording failures and learning from them.

At the moment, we are in the process of identifying what kind of data might be particularly useful in a failsafe context and are investigating the different types of data that we believe might be useful for this purpose in greater detail. After collecting fail-safe data from different sources in a range of environmental situations, we are planning to use this data to automatically create LSMs and a GSM. Our PdF systems will be based on the MindSphere ${ }^{2}$ platform, an industrial cloud-based platform that is provided by Siemens $\mathrm{AG}$, one of the largest industrial automation companies.

\section{See https://siemens.mindsphere.io/}

This mitigates data security and privacy issues, as these challenges are already taken care of by the MindSphere platform. To connect industrial deployments, Siemens provides a specific class of devices - called MindConnect - that takes care of the secure data transfer from PLCs. These fail-safe modules, in turn, can be used to access the raw fail-safe data discussed in Section 3.1. After getting access to the necessary data, we will create applications that run on top of the MindSphere platform and create LSMs and GSMs that can be used for data analysis and to extract conclusions about the (predicted) fail-safe state of the observed system.

\section{Conclusion}

This project is at an early stage and there is a lot of work ahead of us for accomplishing our final goal of creating a demonstrator of a Predictive Fail-Safe system that can improve the safety of an industrial automation system and its environment and support the system in rapidly adapting to fail-safe-relevant dynamics. Observing an industrial environment, we noticed a large number of currently unused data sources that we expect to be relevant to future PdF systems. In this paper, we have collected and classified these sources and have outlined how we intend to use them in the context of creating local and global models of fail-safe relevant aspects of automation systems.

We believe that the domain of industrial fail-safe can profit from the combination of machine learning approaches with data from industrial fail-safe systems and environmental data that we can obtain through connected sensors in the surroundings of an industrial automation system. At the same time, we expect such a system to be able to handle increasingly dynamic environments in smart factories, thereby enabling the faster reconfiguration of fail-safe systems.

\section{References}

[1] D. Evans, "The internet of things: How the next evolution of the internet is changing everything," Cisco Internet Business Solutions Group (IBSG), Tech. Rep., 2011.

[2] D. Gorecky, M. Schmitt, M. Loskyll, and D. Zühlke, "Human-machineinteraction in the industry 4.0 era," in Proc. INDIN, 2014, pp. 289-294.

[3] I. H. Witten, E. Frank, M. A. Hall, and C. J. Pal, Data Mining: Practical Machine Learning Tools and Techniques, 4th ed. Morgan Kaufmann, 2017.

[4] M. Hofmann, F. Neukart, and T. Bäck, "Artificial intelligence and data science in the automotive industry," Comp. Research Repository, 2017.

[5] H. M. Hashemian, "State-of-the-art predictive maintenance techniques," IEEE Trans. Instrum. Meas., vol. 60, no. 1, pp. 226-236, 2011.

[6] R. K. Mobley, "Benefits of predictive maintenance," in An Introduction to Predictive Maintenance, 2nd ed., ser. Plant Engineering, R. K. Mobley, Ed. Butterworth-Heinemann, 2002, pp. 60-73.

[7] H. Bauer, C. Baur, G. Camplone, and K. G. et al., "Industry 4.0 how to navigate digitization of the manufacturing sector," McKinsey Industry 4.0 Global Expert Survey, Tech. Rep., 2015.

[8] S. Mayer, D. Plangger, F. Michahelles, and S. Rothfuss, "UberManufacturing: A goal-driven collaborative industrial manufacturing marketplace," in Proc. IoT. ACM, 2016, pp. 111-119. 